\title{
The impact of pre- and post-natal contexts on immunity, glucocorticoids and oxidative stress resistance in wild and domesticated grey partridges
}

\author{
Benjamin Homberger*,1,2, Susanne Jenni-Eiermann ${ }^{1}$, Alexandre Roulin ${ }^{3}$ and Lukas Jenni ${ }^{1}$ \\ ${ }^{1}$ Swiss Ornithological Institute, Seerose 1, 6204 Sempach, Switzerland; ${ }^{2}$ Institute of Evolutionary Biology and \\ Environmental Studies, University of Zurich, 8057 Zurich, Switzerland; and ${ }^{3}$ Department of Ecology and Evolution, \\ Biophore, University of Lausanne, 1015 Lausanne, Switzerland
}

\begin{abstract}
Summary
1. Genetic background, prenatal and post-natal early-life conditions influence the development of interconnected physiological systems and thereby shape the phenotype. Certain combinations of genotypes and pre- and post-natal conditions may provide higher fitness in a specific environmental context.
\end{abstract}

2. Here, we investigated how grey partridges Perdix perdix of two strains (wild and domesticated) cope physiologically with pre- and post-natal predictable vs. unpredictable food supply. Food unpredictability occurs frequently in wild environments and requires physiological and behavioural adjustments.

3. Well-orchestrated and efficient physiological systems are presumably more vital in a wild environment as compared to captivity. We thus predicted that wild-strain grey partridges have a stronger immunity, glucocorticoid (GC) stress response and oxidative stress resistance (OSR) than domesticated birds, which have undergone adaptations to captivity. We also predicted that wild-strain birds react more strongly to environmental stimuli and, when faced with harsh prenatal conditions, are better able to prepare their offspring for similarly poor post-natal conditions than birds of domesticated origin.

4. We found that wild-strain offspring were physiologically better prepared for stressful situations as compared to the domesticated strain. They had a high GC stress response and a high OSR when kept under predictable food supply. Wild-strain parents reacted to prenatal unpredictable food supply by lowering their offspring's GC stress response, which potentially lowered GC-induced oxidative pressure. No such pattern was evident in the domesticated birds.

5. Irrespective of strain and prenatal feeding scheme, post-natal unpredictable food supply boosted immune indices, and GC stress response was negatively related to antibody response in females and to mitochondrial superoxide production.

6. Wild-strain grey partridge showed fitness-relevant physiological advantages and appeared to prepare their offspring for the prospective environment. Negative relationships between GC stress response, immunity and oxidative indices imply a pivotal role of an organism's oxidative balance and support the importance of considering multiple physiological systems simultaneously.

Key-words: animal re-introduction, domestication effects, glucocorticoid stress response, immunocompetence, maternal effects, mitochondrial superoxide production, oxidative balance, oxidative stress resistance, physiological networks, trade-off

\section{Introduction}

Investigating the sources and consequences of phenotypic variation is central for understanding the evolution of

*Correspondence author. E-mail: beni.homberger@vogelwarte.ch life-history strategies and important for the conservation of threatened species (Ricklefs \& Wikelski 2002). Three major factors contribute to shape the phenotype, namely the genetic background, the prenatal parental environmental and the post-natal early-life environmental conditions. 
These factors can interact with each other and provide the physiological framework on which phenotypic variation is based (e.g. Love et al. 2008; Henriksen, Rettenbacher \& Groothuis 2011; Cohen et al. 2012).

Genetic variation provides the raw material for selection and partly determines the evolutionary potential of a species. Considering the genetic origin is especially important in the context of animal re-introductions using captive populations. Reduced genetic diversity (Keller et al. 2012) and adaptations to captivity, which are detrimental in the wild (Frankham 2008), could compromise the success of animal re-introductions using captive stocks (Fischer \& Lindenmayer 2000).

In addition to the genetic make-up, the phenotype is shaped by parental effects (Mousseau \& Fox 1998; Breuner 2008; Love \& Williams 2008a; Coslovsky \& Richner 2011). Prenatal maternal effects (i.e. during the egg-laying period or gestation) can be seen as a mechanism to adapt the offspring to the prospective environment the mother experienced before or during pregnancy/gestation or egg laying (Mousseau \& Fox 1998; Love \& Williams 2008b). This may be a direct consequence of limited resources that, for example, increase maternal glucocorticoids (GCs) that in turn are transferred proportionally to the embryo and prepare it to harsh conditions (Breuner 2008; Love \& Williams 2008a). It may also be a regulated transfer of hormones (e.g. androgens) depending on environmental and maternal conditions (Groothuis \& Schwabl 2008).

Post-natal early-life conditions, the third major factor shaping the phenotype, have been shown to induce lasting effects on an individual's phenotypic expression and can consequently affect fitness (Metcalfe \& Monaghan 2001; Tschirren et al. 2009). Thus, a certain combination of genetic background, prenatal and early-life conditions may shape the offspring's phenotype to suit a specific environment.

Phenotypic variation emerges from a complex interplay of physiological systems and the environment (Cohen et al. 2012). These physiological interactions and environmental constraints may entail physiological trades-offs, which in turn might constrain phenotypic expression (Ricklefs \& Wikelski 2002). Physiological systems that were shown to be affected by environmental conditions include the GC stress response (the excretion of GCs as a response to stress by the hypothalamic-pituitary-adrenal axis; Love \& Williams 2008b) and the immune system (Tschirren et al. 2009). The GC excreting system and the immune system are interconnected in such a way that high levels of GCs often down-regulate immune reactivity (Sapolsky, Romero \& Munck 2000; but see Martin 2009). Thus, there appears to be a trade-off between immunity and GC stress response in that a high activity of one system constrains the activity of the other. An associated cost of both, the immune system and GC stress response, is the production of reactive oxygen species (ROS; Knight 2000; Costantini \& Møller 2009). ROS are highly reactive molecules, which are ubiquitous in aerobic organisms (Dowling \& Simmons 2009).
While ROS support vital functions such as pathogen destruction in an immune response (Knight 2000), they also pose an oxidative threat that has to be tightly controlled to minimize indiscriminate damage to the cells (Monaghan, Metcalfe \& Torres 2009). Therefore, an organism possesses an arsenal of enzymatic and non-enzymatic antioxidants that maintain oxidative homoeostasis under normal conditions. However, if the oxidative load exceeds the capacity of the antioxidant system, the balance is shifted towards ROS resulting in oxidative stress and its malign consequences to the host cell. In recent years, ROS have repeatedly been suggested as important players in the evolution of life-history strategies (Dowling \& Simmons 2009; Monaghan, Metcalfe \& Torres 2009; Metcalfe \& Alonso-Alvarez 2010), and they could constrain the expression of different phenotypes. Because the production of ROS can increase drastically within a short time-scale [e.g. due to intense physical activity, immune challenge and GC stress response (Knight 2000; Sachdev \& Davies 2008; Costantini, Marasco \& Møller 2011)], there must normally be an excess of antioxidant capacity as a contingency buffer (Monaghan, Metcalfe \& Torres 2009), which is called oxidative stress resistance (OSR). Thus, OSR is the remaining current capacity to prevent oxidative stress, which integrates past and present oxidizing events with regard to all antioxidants available (Lesgards et al. 2002; Stocker et al. 2003). Another important index of the oxidative balance is mitochondrial superoxide (mSO) production. Mitochondria are responsible for the majority of ROS generation (Balaban, Nemoto \& Finkel 2005), and superoxide is the principal ROS produced (Turrens 1997; Mukhopadhyay et al. 2007).

In the present study, we controlled prenatal and earlylife food availability of two strains of grey partridges ( Perdix perdix; Fig. 1). Food availability is a crucial factor for wild birds, affecting, for example, timing of reproduction (Schoech \& Hahn 2008) or offspring sex ratio (Merkling et al. 2012). Unpredictable or restricted access to food can affect immune function (Alonso-Alvarez \& Tella 2001) and provoke physiological changes such as weight loss and/or increased levels of circulating GCs in birds (Lynn et al. 2010). Circulating levels of GCs in female birds during egg laying can be correlated with GC levels in the egg and affect the offspring's stress axis (Love, McGowan \& Sheriff 2012). GCs could also affect the offspring by modulating levels of other egg hormones (e.g. high circulating maternal GCs entailed lower levels of yolk progesterone and testosterone; Henriksen, Groothuis \& Rettenbacher 2011).

We took advantage of a re-introduction project of grey partridges into Switzerland (Jenny, Holzgang \& Zbinden 2005), which used recent descendants of wild birds vs. birds bred in captivity for more than 30 generations. The two strains were predicted to differ by the degree of domestication. By combining the two strains with pre- and post-natal food treatments, we created eight experimental groups. We determined the impact of strain, pre- and postnatal food availability on indices of the innate and 


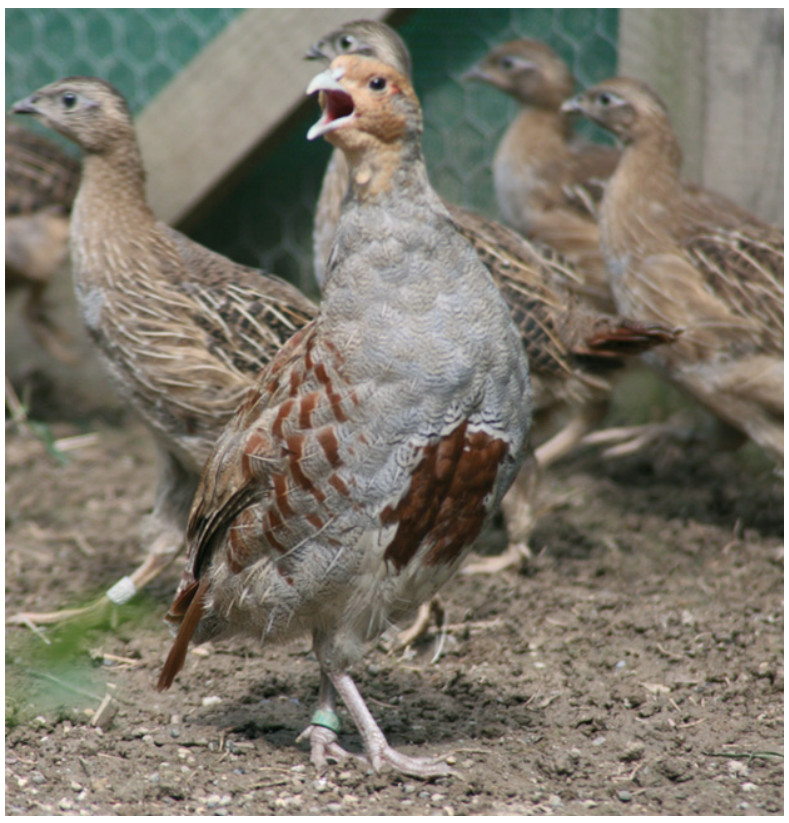

Fig. 1. Adult (foreground) and juvenile (around 3 weeks old, background) grey partridges in one of the outdoor aviaries in Sempach, Switzerland. Photograph by B. Homberger.

adaptive immune system, on OSR, on mSO production and on the GC stress response. These physiological systems are interconnected, and all represent considerable intrinsic sources of ROS, which must be balanced (Costantini \& Møller 2009; Stier et al. 2009; Costantini, Marasco \& Møller 2011). Thus, we investigated whether there are correlations between the systems, which may be indicative of trade-offs in regard to oxidative balance.

Our first aim in this study was to investigate the impact of moderate stress (experienced as unpredictable food supply during crucial time frames) prenatally and post-natally early in life on important physiological traits that underlie phenotypic variation. We were especially interested in potential multiplicative effects of pre- and post-natal treatment in the two strains. The second aim was to examine the interconnected nature of the physiological traits with regard to their oxidative potential.

We predicted that wild-strain grey partridges have a stronger immunity, GC stress response and OSR than domesticated birds, which have undergone adaptations to captivity (Price 1999). We hypothesized that unpredictable food supply represented a latent challenging situation, which might provoke physiological adaptations. Mothers experiencing unpredictable food supply might prepare their offspring to the expected demanding environmental conditions, while unprepared offspring (i.e. offspring that was not prenatally subjected to unpredictable food supply) might suffer from an inappropriate physiological set-up (Price 1999; Breuner 2008). The OSR could play an important role because stress physiology, immunity and mitochondrial activity represent intrinsic sources of ROS. To our knowledge, this is one of the first studies trying to manipulate three important sources of phenotypic variation and characterizing their consequences on important physiological traits in a non-model species.

\section{Materials and methods}

\section{ORIGIN OF BIRDS}

The grey partridge is a ground-dwelling, game bird species typically inhabiting farmland areas. They have precocial young, are seasonally monogamous and lay normally one clutch per year. In 2010, grey partridge eggs were obtained from a breeder in the UK (Perdix Wildlife Solutions, Warwickshire, UK). Eggs originated from two captive strains. For the first strain, male and female grey partridges were captured from a sustainable wild population on a large wild-game shooting estate in eastern England. Female offspring from these wild pairs were mated with males captured in the wild the consecutive spring. Offspring of this semi-wild strain were subjected to prenatal treatment and produced part of the eggs for our study. Thus, these birds are considered to be genetically similar to the wild population (subsequently called wild strain). The second strain consisted of birds that were kept in captivity for at least 30 generations (one generation per year) without adding new wild birds (subsequently called domesticated strain). The captive environment was believed to have caused random genetic changes and/or adaptations to captivity as they occur during the domestication process (Price 1999; Keller et al. 2012). A pilot study found significant differences in polymorphic satellite markers between the two strains, but no signs of inbreeding in the domesticated strain (B. Homberger, unpublished data). During winter, wild and domesticated birds were held in two separate aviaries adjacent to each other on the same farm in the UK (Perdix Wildlife Solutions)

\section{EXPERIMENTAL PROCEDURE}

When mating season started, birds were allowed to freely choose their mate from the flock with which they spent the winter. Twenty-five pairs of wild and 25 pairs of domesticated grey partridges were then placed into separate outdoor aviaries $(3 \times 3 \times 1.5 \mathrm{~m})$ and randomly assigned to either predictable or unpredictable food supply. In the predictable food supply scheme (12 pairs of each strain), food was available ad libitum $24 \mathrm{~h}$ a day. In the unpredictable food supply scheme (13 pairs of each strain), food was removed during $4 \mathrm{~h}$ a day at randomly differing time points (between 8 am and $8 \mathrm{pm}$ ) in accordance with the UK regular feeding schemes, while during the remaining time, food was available ad libitum. The unpredictable feeding scheme began on 5 April 2010, that is, 1 week before egg laying began and lasted throughout the entire egg-laying period until the last series of eggs was transported to Switzerland at the end of June 2010. Grey partridge hens laid 42 eggs on average (no significant difference between feeding schemes). We found that eggs of parents subjected to prenatal unpredictable food supply had a lower progesterone concentration in yolk than eggs of parents subjected to prenatal predictable food supply, while GC and testosterone levels were similar in the two groups of eggs (B. Homberger, unpublished data). This suggests that the food supply scheme altered hormonal maternal effects.

Eggs were collected daily from the aviaries and transported weekly by airplane to Switzerland. They were artificially incubated at the Swiss Ornithological Institute facilities in Sempach. On the hatching day, chicks were individually marked with colour rings and assigned to indoor aviaries $(200 \times 80 \times 80 \mathrm{~cm})$ in Sempach with c. 30 birds of the same hatching day per aviary. Indoor aviary groups consisted of an equal number of birds (i.e. 7 or 8 ) per strain $\times$ prenatal treatment combination. The first week 
post-hatch, food (Trutenküken Vormast; Kliba Nafag, Kaiseraugst, Switzerland) and water were provided ad libitum. From day 7 to day 29 post-hatch, the post-natal feeding scheme was conducted, which consisted of food and water withdrawal during $3 \mathrm{~h}$ a day at randomly varying time points between $8 \mathrm{am}$ and $8 \mathrm{pm}$ (in contrast to the parents held in outdoor aviaries, water withdrawal was feasible for the chicks in the indoor aviaries and adds to the unpredictability of resource availability; Levine \& Ursin 1991). The birds that were allocated to post-natal predictable food supply received food and water ad libitum throughout the post-natal treatment phase. On day 29 , the chicks were transferred into outdoor aviaries in Sempach $(8 \times 4 \times 2 \mathrm{~m})$ and were rearranged so that the new groups consisted of an equal number of birds of each strain $\times$ prenatal $\times$ post-natal food treatment combination (approximately four birds per combination) and of similar group size (approximately 32 birds per aviary group in total). Outdoor aviaries included hideaways of coniferous branches, sand-bathing opportunities and natural grassy vegetation. Food and water were provided ad libitum all day. Throughout the study, all aviaries were visited equally frequently.

Body mass at hatching was higher in domesticated strain chicks (mean hatching body mass domesticated $=10.1 \mathrm{~g} \pm 0.09 \mathrm{SE}$; wild $9.4 \mathrm{~g} \pm 0.07 \mathrm{SE} ; \chi^{2}=12.14$, d.f. $\left.=1, P<0.001\right)$, but did not differ between prenatal treatments $\left(\chi^{2}=1.85\right.$, d.f. $\left.=1, P>0 \cdot 1\right)$. On day 55 after hatching (the day of blood sampling and vaccination), body mass did not differ between strain $\times$ prenatal $\times$ postnatal food treatment combinations $\left(\chi^{2}=5.84\right.$, d.f. $\left.=7, P=0.55\right)$ but tended to be higher for males (mean body mass males $=234.3 \mathrm{~g} \pm 2.5 \mathrm{SE} ;$ females $225.5 \mathrm{~g} \pm 2.8 \mathrm{SE} ; \chi^{2}=3.03$, d.f. $=1, P=0 \cdot 08)$.

\section{SAMPLING PROTOCOL}

This section gives an overview of the sampling procedure. Methodological details are given in the following sections. In total, we raised 1360 chicks in 2010. A random subsample of 200 individuals (plus 48 control birds for the vaccination experiment) from 13 aviary groups was used for this study (25 birds of each strain $\times$ prenatal $\times$ post-natal food treatment combination). On day 55 after hatching, these birds were blood-sampled twice within $30 \mathrm{~min}$ by puncturing the brachial vein (about $100 \mu \mathrm{L}$ blood). The first blood sample was taken to measure baseline level of corticosterone, the primary GC in birds. From the second sample, we determined the GC stress response to an acute stressor, antibody baseline values (measurement of adaptive immunity without prior immune challenge), innate humoural immunity, OSR and $\mathrm{mSO}$ production. On the same day, these birds were injected with an immune stimulant to provoke a response of the adaptive immune system and an additional 48 with a control agent. Eight days after injection (day 63 after hatching), control and vaccinated birds were blood-sampled again to measure antibody response (measurement of adaptive immunity after immune challenge). Either blood samples were analysed within $8 \mathrm{~h}$ (OSR, mSO production) or plasma was stored at $-20{ }^{\circ} \mathrm{C}$ until analysis (agglutination, antibody response and corticosterone). Sample size per parameter varied (see respective tables), because we could not obtain measurements of all indices for all individuals (in particular, GC measurement was restricted to the 166 individuals blood-sampled within $3 \mathrm{~min}$ ).

\section{DETERMINATION OF THE BASELINE CORTICOSTERONE AND GC STRESS RESPONSE}

Baseline- and stress-induced levels of corticosterone could be assessed in 166 birds. Baseline samples were obtained within $3 \mathrm{~min}$ after entering the aviary (first disturbance) and the GC stress response after $30 \mathrm{~min}$ of restraint in a cotton bag. Corticosterone level was determined using an enzyme immunoassay (Munro \& Stabenfeldt 1984). In short, corticosterone was extracted from plasma using $4 \mathrm{~mL}$ dichloromethane and incubated overnight allowing adherence of corticosterone onto the well surface linked by the antibody (Chemicon; cross-reactivity: 11 -dehydrocorticosterone $0.35 \%$, progesterone $0.004 \% \quad 18$ hydroxydeoxycorticosterone $0.01 \%$, cortisol $0.12 \%$, 18-hydroxycorticosterone $0.02 \%$ and aldosterone $0.06 \%$ ). A horse radish peroxidase-corticosterone complex served as enzyme label and 2,2azino-bis(3-ethylbenzthiazoline-6-sulphonic acid) (ABTS) as substrate. Samples were evaluated in triplicates. Coefficients of variance were $2.5 \%$ and $22 \%$ within and between plates, respectively. For a detailed description of the assay, see Mueller et al. (2007).

\section{DETERMINATION OF OSR}

Oxidative stress resistance was determined in 212 birds (200 individuals plus 12 from the PBS control group) using the KRL test ('SPIRAL' laboratories, Couternon, France) that was derived from human medicine and adapted to bird physiology (AlonsoAlvarez et al. 2004). The test measures OSR in a whole blood sample, especially erythrocyte membrane resistance to oxidants, which is tightly linked to vitamin E (Stocker et al. 2003; Monaghan, Metcalfe \& Torres 2009). The test has repeatedly been used to evaluate global antioxidant capacity including enzymatic and non-enzymatic antioxidants (Lesgards et al. 2002; Stocker et al. 2003; Bize et al. 2008).

One hundred and eighty microlitre of the KRL/blood solution was subjected to a controlled attack of ROS [2, 2'-azobis-(aminodinopropane) hydrochloride (AAPH)]. After initiating the ROS attack, the optical density (OD), that is, consecutive cell densities in the sample, was repeatedly measured at $540 \mathrm{~nm}$ in $90 \mathrm{~s}$ bouts at $37{ }^{\circ} \mathrm{C}$ to determine the time needed to haemolyse $50 \%$ of the red blood cells in the sample. Initial OD, that is, the initial density of cells in the sample, was assessed prior to the start of the assay and appeared to be an important predictor of haemolysis time.

\section{DETERMINATION OF ADAPTIVE HUMOURAL IMMUNITY}

To determine an individual's ability to mount a specific immune response, we conducted a vaccination assay. Two hundred birds were injected with $100 \mu \mathrm{L}$ TETRAVAC vaccine (Sanofi Pasteur SA, Lyon, France), a polyvalent vaccine against diphtheria, tetanus, pertussis and poliomyelitis used for human vaccination and used to induce antibody production in birds (Gasparini et al. 2009; Stier et al. 2009). An additional 48 birds from the same 13 aviary groups representing all strain $\times$ prenatal $\times$ post-natal food treatment combinations were treated the same (handling procedures and feeding schemes) but received a $100 \mu \mathrm{L}$ PBS (P3813; Sigma-Aldrich, Buchs, Switzerland) injection as control. Injected birds were recaptured 8 days after vaccination, and a second blood sample was taken to determine the antibody response.

To determine antibody titre before and after TETRAVAC or PBS injection, we used a sandwich ELISA as described by Gasparini et al. (2009). Microtitre plates were coated with $100 \mu \mathrm{L}$ of $1: 50$ diluted TETRAVAC vaccine for $2 \mathrm{~h}$ at room temperature. Plates were washed with PBS containing $0.05 \%$ Tween (PBS-Tween, Tween 20; Reactolab, Servion, Switzerland), and unspecific binding sites were covered with PBS-Tween containing 5\% milk (PBS-milk, blotting grade blocker non-fat dry milk; Bio$\mathrm{Rad}$, Reinach, Switzerland) for $2 \mathrm{~h}$ at room temperature. After washing the plates, they were mounted with $100 \mu \mathrm{L}$ diluted plasma $(1: 80)$ and incubated overnight at $4{ }^{\circ} \mathrm{C}$. The following morning, plates were washed and equipped with $100 \mu \mathrm{L}$ of 
peroxidase-conjugated rabbit anti-chicken IgG (A-9046; SigmaAldrich) diluted in PBS-milk $(1: 3000)$ and incubated for $2 \mathrm{~h}$ at room temperature. Plates were washed, and $100 \mu \mathrm{L}$ of peroxidase substrate (o-phenylenediamine dihydrochlorides; Sigma-Aldrich) was added for $15 \mathrm{~min}$ at room temperature. Finally, the reaction was stopped using $50 \mu \mathrm{L}$ of hydrochloric acid ( $\mathrm{HCl} 1 \mathrm{M})$, and the OD was measured at 490 -nm wavelength using a photo-spectrometer (Wallac Victor 1420; Perkin Elmer LAS, Rodgau-Jügesheim, Germany). All samples were evaluated in triplicates and calibrated according to a standard consisting of four serial dilutions of a positive pool sample $(1: 50,1: 80,1: 100,1: 500)$. Repeatability within and between plates was high (coefficients of variance $4.5 \%$ and $11.7 \%$, respectively).

\section{DETERMINATION OF INNATE HUMOURAL IMMUNITY}

To estimate an individual's potential to mount an unspecific, innate immune response, we measured haemolysis and haemagglutination in 167 birds according to Matson, Ricklefs \& Klasing (2005) with small modifications of the dilutions. Serial dilutions of plasma with PBS were prepared in 96-well round-bottom assay plates, and $1 \%$ rabbit red blood cell suspension was added. Column one contained $20 \mu \mathrm{L}$ pure plasma, whereas columns 2-11 were equipped with $20 \mu \mathrm{L}$ of PBS. Twenty microlitre of plasma was added to column two and mixed, and $20 \mu \mathrm{L}$ of the dilution was transferred to column 3 and so on. Hence, we tested serial dilutions of plasma ranging from 1 (pure plasma) to 1024. Column 12 contained PBS only as a negative control. Twenty microlitre of $1 \%$ rabbit red blood cell suspension (Harlan Laboratories, An Venray, the Netherlands) was added to all wells. Plates were sealed, tilted and incubated at $37^{\circ} \mathrm{C}$ for $90 \mathrm{~min}$. Plates were then photographed twice using a high-resolution digital camera (Finepix S100; Fujifilm, Tokyo, Japan), and haemolysis and haemagglutination were scored according to Matson, Ricklefs \& Klasing (2005).

\section{DETERMINATION OF MSO PRODUCTION}

We measured mSO production for 210 birds (200 individuals plus 10 from the PBS control group) with the flow cytometric assay described by Mukhopadhyay et al. (2007) and Olsson et al. (2008). We used a molecular probe (MitoSox Red, M36008; http://www.invitrogen.com) that is oxidized specifically by superoxides but not by other ROS within the mitochondria. Sixteen microlitre of whole blood was diluted with $584 \mu \mathrm{L}$ KRL buffer $\left(150 \mathrm{~mm} \mathrm{Na}{ }^{+}, 120 \mathrm{~mm} \mathrm{Cl}-, 6 \mathrm{~mm} \mathrm{~K}{ }^{+}, 24 \mathrm{~mm} \mathrm{HCO}_{3}^{-}, 2 \mathrm{~mm}\right.$ $\mathrm{Ca}_{2}^{+}, 340$ mOsmol L ${ }^{-1}, \mathrm{pH}$ 7.4) immediately after blood sampling. Four hundred and four microlitre of this solution was washed and dosed with $1 \mu \mathrm{L}$ of $0.5 \mathrm{~mm}$ MitoSox. The rest of the solution was used in the analysis of OSR. After an incubation period of $35 \mathrm{~min}$ $($ mean $=35.5 \mathrm{~min} \pm 2.9 \mathrm{SD})$ at $37^{\circ} \mathrm{C}, \mathrm{mSO}$ generation was measured using FACSCalibur flow cytometer and Cell Quest Pro software (BD Biosciences, http://www.bdbiosciences.com). Median fluorescence for a total of 50000 cells per sample was determined and used for statistical analysis. Because time latency between incubation start and measurement can influence measurements and varied slightly between the samples, it was included as a covariate in the analysis.

\section{DATA ANALYSIS}

We built mixed-effect models with identity link function for each of the six following dependent variables: GC stress response (corticosterone levels after $30 \mathrm{~min}$ of restraint), adaptive immunity (antibody response to vaccination), innate immunity (haemagglutination and haemolysis scores), mSO production and OSR. The dependent variables were power-transformed (Box \& Cox 1964) if needed to suffice statistical assumptions. Prior to model selection, all starting models included the following independent variables: strain (wild, domesticated), pre- and post-natal feeding scheme as main effects and up to the three-way interaction; sex as main effect and in interaction with strain, pre- and post-natal feeding scheme (two-way interactions); body condition (residual body mass corrected for tarsus length); sampling date. Body condition and sampling date never proved to be significant and thus do not appear in the tables of the final models. Parental pair ID was included as random effect in all models. For the specific models, we also included the following confounding variables: for the analysis of GC stress response, we included GC baseline (fixed effect) and aviary group (random effect); in the analysis of OSR, we included the initial OD (fixed effect) and laboratory series (random effect); in the analysis of adaptive immunity, we included the type of vaccination (PBS control or TETRAVAC) as fixed effect and laboratory series (random effect); in the analysis of innate immunity, we included laboratory series (random effect); in the analysis of $\mathrm{mSO}$, we included the latency between incubation start and measurement (fixed effect) and laboratory series (random effect).

To account for the interconnected nature of the physiological systems, we added in a second step the six other target variables (GC stress response, OSR, antibody response, agglutination score, lysis score and $\mathrm{mSO}$ ) as main effects and in interaction with sex. Including predictor variables that might themselves be affected by the experimental treatment can conceal the potential effects of the treatment. Whenever there was a significant effect of one of these additional independent terms, we thus indicate whether and to what degree significance of the main terms (strain, pre- and post-natal food supply) changed.

All analyses were conducted using the generalized mixed-effect procedure provided by the lme4 package (Bates, Maechler \& Bolker 2012) in R 2.15 (R Development Core Team 2012). For the mixed model estimates, $P$-values based on the chi-square statistic were calculated using likelihood ratio tests (Bolker et al. 2009). We performed stepwise backwards model selection eliminating non-significant terms and performed likelihood ratio tests for each variable while leaving all other predictors in the model. We kept the main treatment predictors (strain, pre- and post-natal food supply) in the final models even if not significant.

For graphical representation and post hoc comparisons, we used a Bayesian approach and simulated joint posterior distributions from the final mixed models using the arm library in $\mathrm{R}$ (Gelman et al. 2012). We conducted post hoc multiple comparisons of factors with more than two levels based on the simulated joint posterior distribution using a mixed model approach (Gelman, Hill \& Yajima 2012). To do so, the final models were refitted, and the factor with the levels to compare was added as random term. The shrinked group mean estimates were then used for multiple comparisons. From the joint posterior distribution of the model parameters, we derived the posterior distribution for each pairwise difference between the means of the factor levels. These posterior distributions were used to obtain the probability of the hypotheses that the pairwise differences were larger than zero $P($ Diff $>0)$. We defined a post hoc comparison to be significant if $P$ (Diff $>0)<0.025$ or $>0.975$ in the case of a two-tailed hypothesis and $P($ Diff $>0)<0.05$ or $>0.95$ in the case of a one-tailed hypothesis.

\section{Results}

\section{GC STRESS RESPONSE}

The GC stress response was significantly affected by the interaction of strain $\times$ prenatal food availability and by 
Table 1. Results of the mixed model analysis of GC stress response and OSR. Significant $P$-values are shown in bold

\begin{tabular}{|c|c|c|c|c|c|c|}
\hline \multirow[b]{2}{*}{ Independent variables } & \multicolumn{3}{|c|}{ GC stress response } & \multicolumn{3}{|c|}{ Oxidative stress resistance } \\
\hline & $\chi^{2}$ & d.f. & $P$ & $\chi^{2}$ & d.f. & $P$ \\
\hline Strain & $16 \cdot 09$ & 1 & $<0.001$ & $5 \cdot 84$ & 1 & 0.016 \\
\hline Prenatal treatment (pre-tr) & 1.56 & 1 & $0 \cdot 21$ & 0.73 & 1 & $0 \cdot 39$ \\
\hline Postnatal treatment (post-tr) & $0 \cdot 23$ & 1 & $0 \cdot 63$ & $2 \cdot 24$ & 1 & $0 \cdot 13$ \\
\hline Sex & & & n.s. & $25 \cdot 54$ & 1 & $<0.001$ \\
\hline Initial cell density & & & n.a. & $12 \cdot 17$ & 1 & $<0.001$ \\
\hline GC baseline & $23 \cdot 06$ & 1 & $<0.001$ & & & n.s. \\
\hline Strain $\times$ pre-tr & $5 \cdot 81$ & 1 & 0.016 & $2 \cdot 45$ & 1 & $0 \cdot 12$ \\
\hline Strain $\times$ post-tr & & & n.s. & $11 \cdot 33$ & 1 & 0.001 \\
\hline Pre-tr $\times$ post-tr & & & n.s. & $0 \cdot 88$ & 1 & $0 \cdot 348$ \\
\hline Strain $\times$ pre $-\operatorname{tr} \times$ post - tr & & & n.s. & 5.84 & 1 & 0.016 \\
\hline
\end{tabular}

GC, glucocorticoid; OSR, oxidative stress resistance; pre-tr, prenatal treatment; post-tr, post-natal treatment; n.a., not applicable; n.s., not significant and removed from the final model.

The effective sample size for the analysis of OSR was $n=212$ and for GC stress response $n=166$. Parental pair ID was included as random effect in both models. Aviary group or laboratory series were added as random effect in the analysis of GC stress response and OSR, respectively.

corticosterone baseline values (Table 1). There was no effect of post-natal unpredictable food supply (Table 1). When testing for the effect of strain alone, it remained strong $\left(\chi^{2}=10.56\right.$, d.f. $\left.=1, P=0.001\right)$ with a higher response for wild than domesticated birds, while there was no effect of prenatal unpredictable food supply alone $\left(\chi^{2}=0.52\right.$, d.f. $\left.=1, P=0.47\right)$.

Wild-strain offspring subjected to pre- and post-natal predictable food supply had a higher GC stress response as compared to the corresponding domesticated birds [Fig. 2a,c; posterior probability $P($ Diff $>0)<0.001$ ]. Wild offspring subjected to prenatal unpredictable food supply had a lower GC stress response as compared to wild offspring in the prenatal predictable supply group [Fig. 2a,b; posterior probability $P($ Diff $>0)=0.02]$. There was no effect of the prenatal treatment within the domesticated group [Fig. 2c,d; posterior probability $P($ Diff $>0)>0 \cdot 1$ ], and there were no significant effects of OSR, innate or adaptive immunity or mSO production (all $P$-values $>0 \cdot 1)$.

\section{OXIDATIVE STRESS RESISTANCE}

Pre- and post-natal treatment affected OSR in the two strains differently resulting in a significant three-way interaction (Table 1). The initial cell density was positively related to OSR (Table 1). However, initial cell density was not related to strain and pre- and post-natal treatments (all $P$-values $>0 \cdot 2$ ). Within all eight strain $\times$ prenatal $\times$ postnatal food treatment groups, males showed higher OSR than females (estimate for males 66.38, SE range 64.77-67.98; females estimate $60 \cdot 1$, SE range 58.44-61.74, Table 1).

Wild-strain offspring subjected to pre- and post-natal predictable food supply had a higher OSR than the corresponding domesticated birds [Fig. 3a,c; posterior probability $P($ Diff $>0)=0 \cdot 046]$. Within the wild strain, prenatal

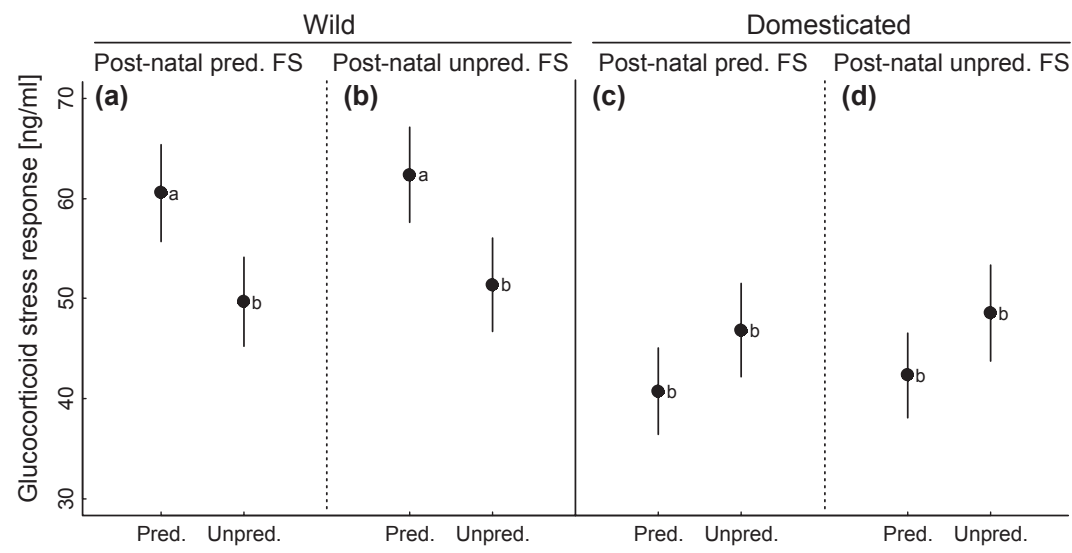

Fig. 2. Predicted means and standard errors for the two strains and the two pre- and post-natal food supply (FS) schemes on glucocorticoid (GC) stress response. The $x$-axis indicates prenatal predictable (pred.) and prenatal unpredictable (unpred.) food supply. (a) Wild offspring, post-natal predictable food supply. (b) Wild offspring, post-natal unpredictable food supply. (c) Domesticated offspring, post-natal predictable food supply. (d) Domesticated offspring, post-natal unpredictable food supply. Predicted means are significantly different from each other $(P<0.05)$ when they do not share the same letters. 


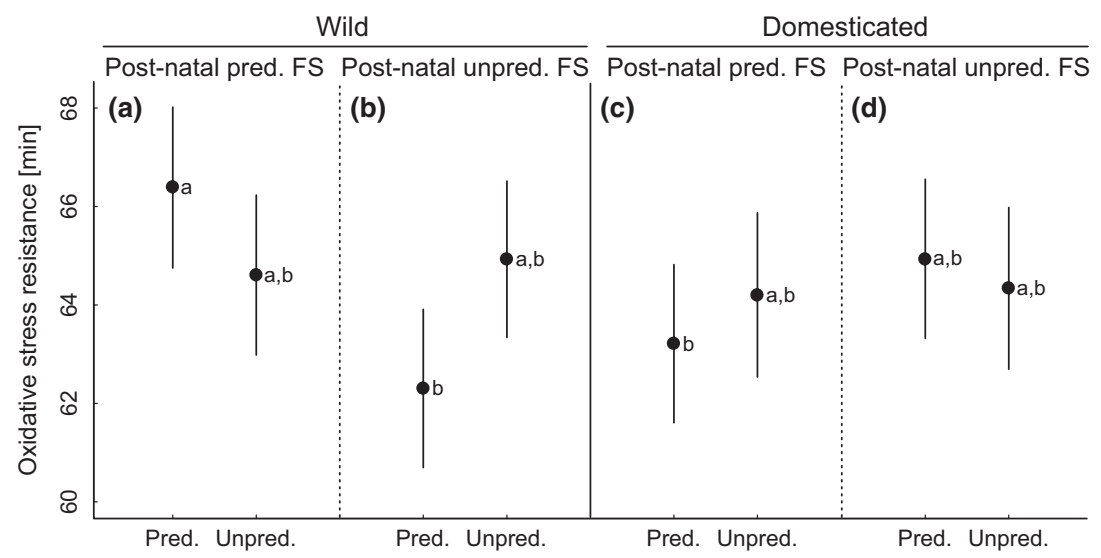

Fig. 3. Predicted means and standard errors for the two strains and the two pre- and post-natal food supply (FS) schemes on oxidative stress resistance (OSR). The $x$-axis indicates prenatal predictable (pred.) and prenatal unpredictable (unpred.) food supply. (a) Wild offspring, post-natal predictable food supply. (b) Wild offspring, post-natal unpredictable food supply. (c) Domesticated offspring, post-natal predictable food supply. (d) Domesticated offspring, post-natal unpredictable food supply. Predicted means are significantly different from each other $(P<0.05)$ when they do not share the same letters.

unpredictable food supply did not markedly affect OSR [Fig. 3a,b; all posterior probability comparisons $P$ $($ Diff $>0)>0 \cdot 1]$. OSR was drastically lower in wild birds encountering prenatal predictable and post-natal unpredictable food supply as compared to pre- and postnatal predictable supply [Fig. 3a,b; posterior probability $P$ (Diff $>0)=0 \cdot 01]$, but OSR was not significantly affected when wild birds encountered both pre- and post-natal unpredictable food supply as compared to pre- and postnatal predictable supply [Fig. 3a,b; posterior probability $P$ $($ Diff $>0)>0 \cdot 1]$. There was no effect of pre- and/or postnatal treatment within the domesticated strain [Fig. 3c,d; all pairwise comparisons within domesticated strain give posterior probabilities $P($ Diff $>0)>0 \cdot 1]$, and there were no significant effects of GC stress response, innate or adaptive immunity or mSO production (all $P$-values $>0 \cdot 1$ ).

\section{ADAPTIVE IMMUNE SYSTEM}

Before stimulation of the adaptive immune system by TETRAVAC vaccination, there were no differences in specific antibody levels between control (PBS-injected) and immunized birds $\left(\chi^{2}=0.11\right.$, d.f. $\left.=1, \quad P=0.74\right)$ and between strain $\times$ prenatal $\times$ post-natal food treatment combinations $\left(\chi^{2}=3.32\right.$, d.f. $\left.=7, P=0 \cdot 85\right)$, but females had higher initial baseline levels of antibodies than males $\left(\chi^{2}=4 \cdot 35\right.$, d.f. $\left.=1, P=0 \cdot 037\right)$. TETRAVAC vaccination successfully elevated antibody production within the same individual (before vaccination mean $=0.0037 \pm 0.0042$ $\mathrm{SD} ; 8$ days after vaccination mean $=0.0331 \pm 0.0178 \mathrm{SD}$; pairwise $t$-test; $t=-22.48$, d.f. $=392, P<0.001$ ), and as a consequence, antibody titre after vaccination differed greatly between vaccinated birds and PBS-injected controls (Table 2). Wild-strain birds and birds subjected to postnatal unpredictable food supply showed a significantly higher antibody response after vaccination compared with the domesticated strain and the predictable supply groups (Fig. 4a, Table 2). Antibody response decreased with
Table 2. Results of the mixed model analysis of the adaptive and innate immunity. Significant $P$-values are shown in bold

\begin{tabular}{|c|c|c|c|c|c|c|}
\hline \multirow[b]{2}{*}{ Independent variables } & \multicolumn{3}{|c|}{ Adaptive immunity } & \multicolumn{3}{|c|}{ Innate immunity } \\
\hline & $\chi^{2}$ & d.f. & $P$ & $\chi^{2}$ & d.f. & $P$ \\
\hline Strain & $5 \cdot 63$ & 1 & 0.018 & 0.94 & 1 & 0.33 \\
\hline Prenatal treatment & $0 \cdot 32$ & 1 & 0.57 & $0 \cdot 00$ & 1 & $0 \cdot 99$ \\
\hline Postnatal treatment & $4 \cdot 25$ & 1 & 0.039 & $5 \cdot 31$ & 1 & 0.021 \\
\hline Sex & 9.79 & 1 & 0.002 & $2 \cdot 21$ & 1 & $0 \cdot 14$ \\
\hline GC stress response & $8 \cdot 52$ & 1 & 0.004 & & & n.s. \\
\hline PBS (placebo) & $30 \cdot 69$ & 1 & $<0.001$ & & & n.a. \\
\hline Strain $\times$ sex & & & n.s. & $7 \cdot 35$ & 1 & 0.007 \\
\hline $\begin{array}{l}\text { GC stress } \\
\text { response } \times \text { sex }\end{array}$ & $7 \cdot 28$ & 1 & 0.003 & & & n.s. \\
\hline
\end{tabular}

GC, glucocorticoid; n.a., not applicable; n.s., not significant and removed from the final model.

The effective sample size for the analysis of adaptive immunity was $n=171$ and innate immunity $n=167$. Parental pair ID and laboratory series were added as random factors in both analyses.

increasing GC stress response in females but not in males (Fig. 5a, Table 2). When omitting GC stress response from the model, effects of strain $\left(\chi^{2}=4.50\right.$, d.f. $\left.=1, P=0.034\right)$ and post-natal food supply remained significant $\left(\chi^{2}=5 \cdot 16\right.$, d.f. $=1, P=0.023)$, and females tended to have a higher antibody response than males $\left(\chi^{2}=3 \cdot 18, \quad\right.$ d.f. $=1$, $P=0.076)$. Prenatal unpredictable food supply did not contribute significantly to explain differences in adaptive immune response irrespective of whether GC stress response was included or not, and there were no significant effects of OSR, innate immunity or mSO production (all $P$-values $>0 \cdot 1)$.

\section{INNATE IMMUNE SYSTEM}

Haemagglutination was significantly higher in birds subjected to post-natal unpredictable food supply compared with birds with a predictable supply (Fig. 4b, Table 2). 

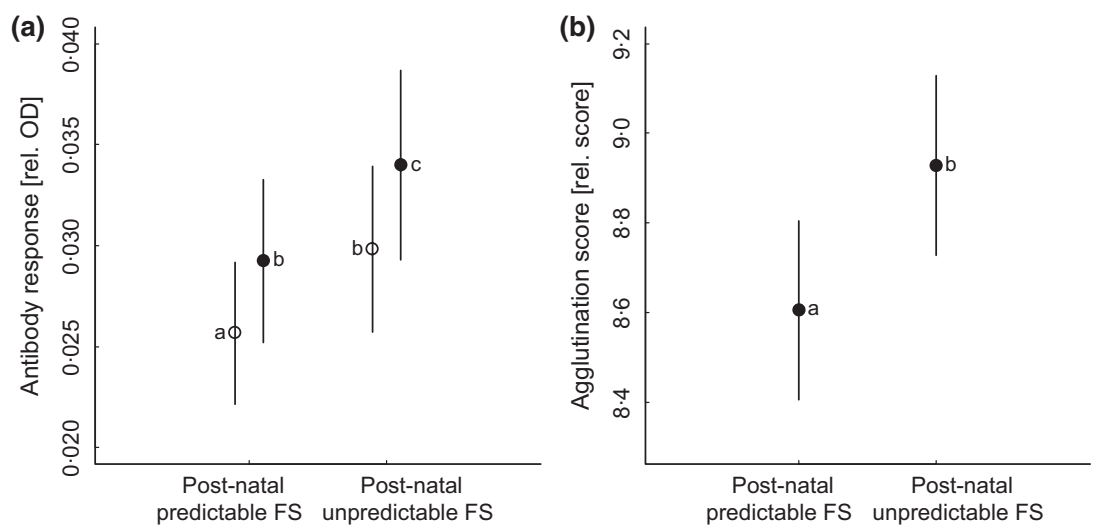

Fig. 4. Predicted means and standard errors for (a) adaptive immunity (antibody response) and (b) innate immunity (agglutination score) in relation to prenatal predictable and post-natal predictable and unpredictable food supply (FS). Open circles represent domesticated strain birds. Filled circles represent wild-strain birds. Prenatal food supply did not have a significant effect. Predicted means are significantly different from each other $(P<0.05)$ when they do not share the same letters.
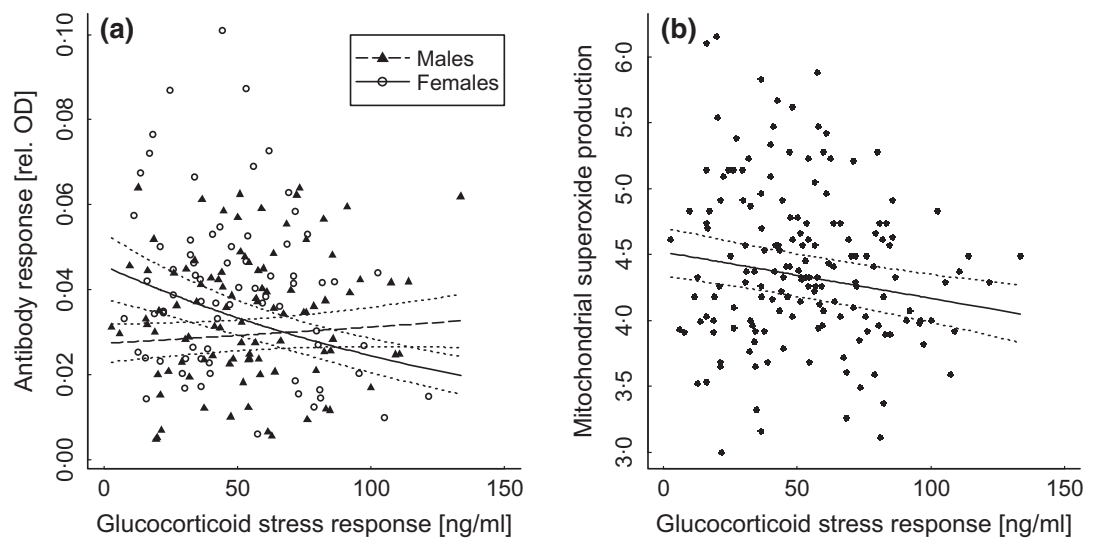

Fig. 5. Raw data points and model estimates with standard errors for the relationships between glucocorticoid (GC) stress response and adaptive immunity (antibody production) (a) and mitochondrial superoxide (mSO) production (b). The panel on the left shows raw data and model estimates for males (triangles, dashed line) and females (open circles, solid line).

There was a significant interaction between strain and sex (Table 2). Domesticated males had lower haemagglutination score than domesticated females (estimate domesticated males $8.06 \pm 0.19 \mathrm{SE}$ and females $8.37 \pm 0.22 \mathrm{SE}$ ), while the opposite was true for wild birds (estimate wild males $8.60 \pm 0.2 \mathrm{SE}$ and females $8.15 \pm 0.21$; Table 2). However, post hoc tests did not reveal significant differences (all posterior probabilities $>0 \cdot 2$ ). There were no significant effects of prenatal food supply, GC stress response, OSR, adaptive immunity and $\mathrm{mSO}$ production on haemagglutination (all $P$-values $>0 \cdot 1$ ).

Similar to haemagglutination, birds subjected to postnatal unpredictable food supply had a higher haemolysis score than birds with a predictable post-natal food supply (estimate for predictable supply $1.98 \pm 0.22 \mathrm{SE}$, estimate unpredictable supply $2.33 \pm 0.22 \mathrm{SE} ; \chi^{2}=4.86$, d.f. $=1$, $P=0.027$ ), but no effects of strain, prenatal food supply or any interaction were evident (all $P$-values $>0 \cdot 1$ ).

In both analyses, there were no effects of GC stress response, OSR, adaptive immunity or $\mathrm{mSO}$ (all $P$-values $>0 \cdot 1)$.

\section{MSO PRODUCTION}

Mitochondrial superoxide production significantly increased with decreasing GC stress response $\left(\chi^{2}=3.93\right.$, d.f. $=1, P=0.048$, Fig. $5 b$ ), but there were no effects of OSR, immune measurements, sex and treatments (main terms and interactions) or any other covariates on $\mathrm{mSO}$ production (all $P$-values $>0 \cdot 1)$. $P$-values for strain and treatment effects were all $>0.4$ irrespective of whether GC stress response was included or omitted from the model.

\section{Discussion}

It appeared that both OSR and GC stress response were affected by strain and prenatal feeding scheme in a similar way. All measures of immune function were boosted by post-natal unpredictable food supply irrespective of strain and prenatal treatment. We also found that GC stress response values in plasma were negatively related to antibody response and $\mathrm{mSO}$, which might highlight the 
regulative role of GCs in the context of resistance to oxidative stress (Fujita et al. 2009; Stier et al. 2009).

\section{EFFECTS OF STRAIN AND FOOD TREATMENTS ON OSR AND GC STRESS RESPONSE}

Birds of the wild strain that were subjected to pre- and post-natal predictable food supply exhibited the highest GC stress response, and at the same time, they had the highest OSR of all groups. Notably, their GC stress response and OSR were markedly higher than those of their domesticated counterparts subjected to pre- and post-natal predictable food supply. This might reflect important consequences of the diverging selective pressures and adaptations under wild or captive conditions. The acute phase of the GC stress response is important to overcome life-threatening situations (Wingfield et al. 1998) and thus can be positively related to fitness (Breuner, Patterson \& Hahn 2008). However, chronically increased GC levels adversely affect fitness traits (Cyr \& Romero 2007) and are related to high levels of oxidative stress (Costantini, Marasco \& Møller 2011). Likewise, high OSR has repeatedly been linked to enhanced fitness in birds from captive (Alonso-Alvarez et al. 2006; Kim et al. 2010b) and wild sources (Bize et al. 2008). We know of no other study comparing OSR of wild and domesticated conspecifics, but heritability of OSR has been found to differ between captive and wild birds. While wild yellow-legged gulls (Larus cachinnans) showed a relatively high heritability of OSR at an age of 8 days ( $h^{2}=0.59$; Kim et al. 2010a), heritability of OSR in zebra finches (Taeniopygia guttata) kept and bred in captivity was low and non-significant at an age of 60 days $\left(h^{2}=0.05 ;\right.$ Kim et al. 2010b). These results imply that there could be a reduced selective pressure on OSR in captivity. However, comparing heritability of OSR in different species living under different conditions can be ambiguous (Costantini \& Verhulst 2009).

We found that the GC stress response of wild offspring was dampened when their parents were subjected to prenatal unpredictable food supply. This might at first sight suggest an adverse effect of the prenatal unpredictable food supply on the phenotype of wild offspring. However, the down-regulation of the stress response could also represent a predictive adaptive response (Breuner 2008; Love \& Williams 2008a), which indicates that the offspring's physiology is being prepared to better match the expected future environment. The adaptive value of such a down-regulation of the GC stress response may include energy saving in an unpredictable or captive environment (Love \& Williams 2008b). We argue that benefits of a down-regulated GC stress response could arise particularly in terms of decreased oxidative load. Indeed, wild birds subjected to post-natal unpredictable food supply but not prenatal unpredictable food supply (thus were not prepared for unpredictable post-natal food supply) showed a strong GC stress response but low OSR. In contrast, wild birds that did encounter prenatal unpredictable food supply (thus were prepared for unpredictable post-natal food supply) exhibited a low GC stress response, and their OSR was insignificantly affected by post-natal unpredictable food supply.

In our case, all birds were subjected to the same frequency of human disturbance, which could elicit a GC stress response. However, OSR of the highly stress-responsive wild birds was only reduced when a high GC stress response coincided with post-natal unpredictable food supply. Predictability is an important factor determining stressfulness of stimuli (Levine \& Ursin 1991). Food unpredictability could enhance the impact of other stressors (Clinchy et al. 2004; Bauer et al. 2011) and/or impair a bird's ability to buffer corticosterone (i.e. reduce the biological activity of corticosterone; Lynn, Breuner \& Wingfield 2003). The combined effect of unpredictable food supply and frequent initiation of a GC stress response in captivity could pose an overwhelming oxidative pressure, which would negatively affect OSR in unprepared birds. Consequently, a prenatal down-regulation of the offspring's GC stress response could alleviate the oxidative threat. The situation could be different in a non-captive context where a strong GC stress response could efficiently help to overcome a real life-threatening situation such as a predator attack (Wingfield et al. 1998).

Given the oxidative burden provoked by GCs (Stier et al. 2009; Costantini, Marasco \& Møller 2011), it makes sense to physiologically couple a strong GC stress response to a strong OSR. Thus, pre- and post-natal permanent food supply enables a high OSR that is capable of absorbing a high GC-induced oxidative load. Therefore, these two traits might have co-evolved through pleiotropic effects or symmorphosis (Ducrest, Keller \& Roulin 2008; Cohen et al. 2012).

In the domesticated strain, no effect of pre- and postnatal treatment on OSR or GC stress response was noticeable. It seems that birds of the domesticated strain do not adapt their chicks to oncoming conditions, perhaps because the GC stress response is reduced anyhow as a result of domestication process (Evans et al. 2006) or they might have lost their ability for subtle fine-tuning (Price 1999). The domestication process leads to profound alterations of physiology and behaviour with detrimental consequences for survival in the wild (McDougall et al. 2006; Frankham 2008). Probably the most important change accompanying the domestication process is a reduction in sensitivity of animals to changes in their environment (Price 1999), which could also include the ability to affect offspring via parental effects.

\section{EFFECTS OF POST-NATAL TREATMENT ON IMMUNITY}

In our study, prenatal unpredictable food supply did not affect immunity, but post-natal unpredictable food supply resulted in a stronger antibody response and a higher haemolysis and haemagglutination score 5 weeks 
after hatch in both strains and sexes. Thus, there appeared to be a lasting effect of post-natal unpredictable food supply early in life on aspects of immunity, which could have important consequences for fitness later on.

Any form of malnutrition (too low or too high food intake, low-quality foods) can impair an adequate immune function (Perez de Heredia, Gomez-Martinez \& Marcos 2012). In laboratory animals where health problems due to excessive food intake are frequent, moderate food restrictions were found to enhance immune function (Fernandes et al. 1997). Here, the positive effects on immunity are likely related to the maintenance of a healthy body condition (Perez de Heredia, Gomez-Martinez \& Marcos 2012). Body condition in our case was not affected by pre-and post-natal treatments and can therefore not explain the observed pattern in immunity. Similar to our findings, other experimental studies in birds revealed that low protein or low total nutritional supply in early life can have lasting enhancing effects on immune indices, which were not solely explained by body condition, but there appear to be further immune-enhancing effects of allegedly adverse early-life conditions (Gonzalez et al. 1999; Tschirren et al. 2009). The lasting boost of immune indices in grey partridges could reflect overcompensation for a reduced immunity during the treatment phase (Birkhead, Fletcher \& Pellatt 1999; Tschirren et al. 2009).

Alternatively, post-natal food unpredictability could have altered the acute stress-induced distribution of immune cells in the body. The acute stress response seems to be important for supporting the redistribution of immune cells from source tissue over the blood stream and into target organs (Dhabhar et al. 2012). During the early acute stress response (up to $30 \mathrm{~min}$ ), lymphocytes (immune cells that are part of the innate and adaptive system) are mobilized from reservoirs into the blood before they enter target tissues. GCs appear to facilitate this redistribution of immune cells out of the blood into the target tissue (Dhabhar et al. 2012). It is difficult to assess how an alteration of the redistribution patterns induced by post-natal unpredictable food supply could have affected an individual's immunocompetence in the field. However, considering acute stress-induced redistribution of immune cells seems to be crucial when investigating immunity, particularly when dealing with highly stress-sensitive wild animals.

\section{CORRELATIONS BETWEEN PHYSIOLOGICAL} PARAMETERS

We found a negative correlation between the activity of the adaptive immune system and the GC stress response in females but not in males. This suggests a potential tradeoff between the adaptive immune system and the GC stress response. Effects of GCs on the immune system are wellknown but appear to be complex and manifold (Sapolsky, Romero \& Munck 2000). In general, GCs at higher con- centrations seem to dampen the activity of the immune system (Klein 2000; Sapolsky, Romero \& Munck 2000), but can differentially affect the innate and the adaptive system (Bourgeon \& Raclot 2006; Stier et al. 2009). Similar to other studies conducted in non-captive species, we found a dampening effect of GCs on the adaptive immune system but no effect on the innate system (Bourgeon \& Raclot 2006; Stier et al. 2009).

Lowering the innate immune system's activity (the first line of defence) in a stressful situation might be too risky, whereas reducing the adaptive system may be a more bearable risk, at least in the short term. In an acute stress state, high levels of GCs could act as a transient down-regulator of a highly active immune system, which could save energy and reduce the risk of oxidative stress and immune overreaction (Sapolsky, Romero \& Munck 2000; Costantini \& Møller 2009; Stier et al. 2009).

We did not find any negative relationship between the adaptive immune system and the GC stress response in males. Sex differences in immune activity have repeatedly been described in many taxa (Nunn et al. 2009) and in relation to the stress physiology (Merrill et al. 2012). Generally, females mount stronger adaptive immune responses than males (Nunn et al. 2009). The strong adaptive immune response provides females with a better protection and may ultimately increase their survival and longevity (Rolff 2002) but also increase the risk of autoimmune disease (Nunn et al. 2009) and oxidative stress (Costantini \& Møller 2009). Indeed, in many species, the incidence of autoimmune disease is higher in females than in males (Klein 2000). A negative feedback, in which high levels of GCs prevent a high antibody response and reduce the risk of autoimmune disease, might be especially valuable in females. On the other hand, further down-regulation of the already moderate antibody response in males might be too dangerous in terms of infection risk. Females had higher baseline values of antibodies than males and tended to mount a stronger antibody response to vaccination. The downside of this pronounced activity of the adaptive immune system might be reflected in a constantly higher oxidative pressure and thus a lower OSR in females than in males, as was found within all strain $\times$ prenatal $\times$ post-natal treatment combinations. Interestingly, other studies have also found sex-specific differences in OSR, which could be due to sex-specific differences in immune system activity (Lesgards et al. 2002; Alonso-Alvarez et al. 2006; Bize et al. 2008). Additionally, in their meta-analysis, Costantini, Marasco \& Møller (2011) concluded that GCs induced a higher oxidative stress in females than in males.

We did not find any noticeable effect of the pre- and post-natal feeding schemes on the $\mathrm{mSO}$ production, but the GC stress response was negatively related to the mSO generation irrespective of treatment, suggesting a second potential trade-off. mSO generation is tightly linked to metabolic rate, oxygen consumption and ATP production (Finkel \& Holbrook 2000). Immediately after encountering a stressor, 
the 'fight and flight' reaction is initiated (Bracha et al. 2004), which up-regulates the metabolic rate and increases oxygen consumption, ATP production and eventually mSO production (Balaban, Nemoto \& Finkel 2005; Costantini, Marasco \& Møller 2011). Hence, both mitochondrial activity and GC stress response represent considerable sources of ROS (Balaban, Nemoto \& Finkel 2005; Costantini, Marasco \& Møller 2011). Maximizing both GC stress response and mitochondrial ATP production might cause an overwhelming oxidative pressure and put an organism at imminent risk of oxidative stress. Two negative feedback loops could theoretically have caused the negative correlation between GC stress response and mSO generation. First, GCs at high concentrations might suppress mSO production. Indeed, it was shown that corticosterone at high but still within physiological relevant concentrations can inhibit mitochondrial ROS generation and ATP production (Fujita et al. 2009). Second, an increase in $\mathrm{mSO}$ production could act as a dampening modulating factor on the expression of a GC response to an acute stressor, similarly as prolonged flight (Jenni-Eiermann et al. 2009) or a reduced body condition (Kitaysky et al. 2005). Both mechanisms could aim to reduce a high cumulative oxidative load but entail the risk of an impaired GC stress response and reduced ATP production, respectively.

\section{Conclusions and perspectives}

This study investigated interactions between genetic set-up (strains), maternal effects (prenatal food availability) and early-life conditions (post-natal food availability) on various physiological parameters of the immune system, GC stress response and oxidative balance in a non-model species.

Wild-strain grey partridges held under predictable food supply conditions showed an array of superior physiological traits indicative of greater overall fitness in the wild. While the wild-strain bird's physiological set-up may not suit a captive environment, they appear to be able to adapt their offspring to captive conditions. Maternal effects could not be shown in the domesticated strain, which could be due to the domestication process. Indeed, a loss of the ability to adaptively shape their offspring to the prospective environment in domesticated strain grey partridges could greatly diminish their fitness in the wild, thus reducing their suitability for re-introduction projects. We plan to further investigate the fitness of our experimental groups in the wild.

It appeared that indices of the immune system were positively affected by post-natal unpredictable food supply. Conducting moderate food restrictions in captivity may provide an easy method to boost an animal's immune function in adulthood, although the method has to be evaluated de novo in each species. As we expect that an enhanced immunity positively affects fitness, this method of rearing may improve re-introduction projects using captive bred individuals.
Finally, we found negative correlations between circulating stress-induced GC levels, adaptive immune response and $\mathrm{mSO}$ production. Investigating physiological networks rather than single aspects of physiology will be crucial for understanding phenotypic expression and its impacts on fitness (Cohen et al. 2012). Our study supports the importance of considering oxidative balance as a key element in the regulation of physiological systems, which could ultimately add to the evolution of life-history strategies.

\section{Acknowledgements}

We thank Pierre Bize for the introduction and assistance when conducing laboratory analysis of OSR and mSO production; Kevin Matson, Kim Stier, Julien Gasparini and Glauco Camenisch for their support when analysing immune indices; David Butler, Imelda Schmid, Christian Dexl and all the assistants who helped us take good care of the birds; and three reviewers for helpful comments on earlier drafts. The project was funded by the Swiss National Science Foundation (grant no 31003A 127057 to LJ and SJ-E). All experiments were carried out under licence of the Veterinary Office of the Canton Lucerne.

\section{References}

Alonso-Alvarez, C. \& Tella, J.L. (2001) Effects of experimental food restriction and body-mass changes on the avian T-cell-mediated immune response. Canadian Journal of Zoology, 79, 101-105.

Alonso-Alvarez, C., Bertrand, S., Devevey, G., Gaillard, M., Prost, J., Faivre, B. \& Sorci, G. (2004) An experimental test of the dose-dependent effect of carotenoids and immune activation on sexual signals and antioxidant activity. The American Naturalist, 164, 651-659.

Alonso-Alvarez, C., Bertrand, S., Devevey, G., Prost, J., Faivre, B., Chastel, O. \& Sorci, G. (2006) An experimental manipulation of life-history trajectories and resistance to oxidative stress. Evolution, 60, 1913-1924.

Balaban, R.S., Nemoto, S. \& Finkel, T. (2005) Mitochondria, oxidants, and aging. Cell, 120, 483-495.

Bates, D., Maechler, M. \& Bolker, B. (2012) lme4: Linear mixed-effects models using S4 classes. R package version 0.999375-42/r1414. Available at: http://R-Forge.R-project.org/projects/lme4/.

Bauer, C.M., Glassman, L.W., Cyr, N.E. \& Romero, L. (2011) Effects of predictable and unpredictable food restriction on the stress response in molting and non-molting European starlings (Sturnus vulgaris). Comparative Biochemistry and Physiology A: Molecular \& Integrative Physiology, 160, 390-399.

Birkhead, T.R., Fletcher, F. \& Pellatt, E.J. (1999) Nestling diet, secondary sexual traits and fitness in the zebra finch. Proceedings of the Royal Society of London B: Biological Sciences, 266, 385-390.

Bize, P., Devevey, G., Monaghan, P., Doligez, B. \& Christe, P. (2008) Fecundity and survival in relation to resistance to oxidative stress in a free-living bird. Ecology, 89, 2584-2593.

Bolker, B.M., Brooks, M.E., Clark, C.J., Geange, S.W., Poulsen, J.R., Stevens, M. \& White, J.S. (2009) Generalized linear mixed models: a practical guide for ecology and evolution. Trends in Ecology \& Evolution, 24, $127-135$.

Bourgeon, S. \& Raclot, T. (2006) Corticosterone selectively decreases humoral immunity in female eiders during incubation. Journal of Experimental Biology, 209, 4957-4965.

Box, G.E.P. \& Cox, D.R. (1964) An analysis of transformations. Journal of the Royal Statistical Society B: Statistical Methodology, 26, 211-252.

Bracha, H.S., Ralston, T.C., Matsukawa, J.M., Williams, A.E. \& Bracha, A.S. (2004) Does "Fight or flight" need updating? Psychosomatics, 45, $448-449$.

Breuner, C. (2008) Maternal stress, glucocorticoids, and the maternal/fetal match hypothesis. Hormones and Behavior, 54, 485-487.

Breuner, C.W., Patterson, S.H. \& Hahn, T.P. (2008) In search of relationships between the acute adrenocortical response and fitness. General and Comparative Endocrinology, 157, 288-295.

Clinchy, M., Zanette, L., Boonstra, R., Wingfield, J.C. \& Smith, J.N.M. (2004) Balancing food and predator pressure induces chronic stress in 
songbirds. Proceedings of the Royal Society of London B: Biological Sciences, 271, 2473-2479.

Cohen, A.A., Martin, L.B., Wingfield, J.C., McWilliams, S.R. \& Dunne, J.A. (2012) Physiological regulatory networks: ecological roles and evolutionary constraints. Trends in Ecology \& Evolution, 27, 428-435.

Coslovsky, M. \& Richner, H. (2011) Predation risk affects offspring growth via maternal effects. Functional Ecology, 25, 878-888.

Costantini, D., Marasco, V. \& Møller, A.P. (2011) A meta-analysis of glucocorticoids as modulators of oxidative stress in vertebrates. Journal of Comparative Physiology B: Biochemical, Systemic, and Environmental Physiology, 181, 447-456.

Costantini, D. \& Møller, A.P. (2009) Does immune response cause oxidative stress in birds? A meta-analysis. Comparative Biochemistry and Physiology Part A: Molecular \& Integrative Physiology, 153, 339-344.

Costantini, D. \& Verhulst, S. (2009) Does high antioxidant capacity indicate low oxidative stress? Functional Ecology, 23, 506-509.

Cyr, N.E. \& Romero, L. (2007) Chronic stress in free-living European starlings reduces corticosterone concentrations and reproductive success. General and Comparative Endocrinology, 151, 82-89.

Dhabhar, F.S., Malarkey, W.B., Neri, E. \& McEwen, B.S. (2012) Stressinduced redistribution of immune cells-From barracks to boulevards to battlefields: a tale of three hormones - Curt Richter Award Winner. Psychoneuroendocrinology, 37, 1345-1368.

Dowling, D.K. \& Simmons, L.W. (2009) Reactive oxygen species as universal constraints in life-history evolution. Proceedings of the Royal Society of London B: Biological Sciences, 276, 1737-1745.

Ducrest, A.L., Keller, L. \& Roulin, A. (2008) Pleiotropy in the melanocortin system, coloration and behavioural syndromes. Trends in Ecology \& Evolution, 23, 502-510.

Evans, M.R., Roberts, M.L., Buchanan, K.L. \& Goldsmith, A.R. (2006) Heritability of corticosterone response and changes in life history traits during selection in the zebra finch. Journal of Evolutionary Biology, 19, 343-352.

Fernandes, G., Venkatraman, J.T., Turturro, A., Attwood, V.G. \& Hart, R.W. (1997) Effect of food restriction on life span and immune functions in long-lived Fischer-344 $\times$ Brown Norway F-1 rats. Journal of Clinical Immunology, 17, 85-95.

Finkel, T. \& Holbrook, N.J. (2000) Oxidants, oxidative stress and the biology of ageing. Nature, 408, 239-247.

Fischer, J. \& Lindenmayer, D.B. (2000) An assessment of the published results of animal relocations. Biological Conservation, 96, 1-11.

Frankham, R. (2008) Genetic adaptation to captivity in species conservation programs. Molecular Ecology, 17, 325-333.

Fujita, C., Ichikawa, F., Teratani, T., Murakami, G., Okada, T., Shinohara, M., Kawato, S. \& Ohta, Y. (2009) Direct effects of corticosterone on ATP production by mitochondria from immortalized hypothalamic GT1-7 neurons. Journal of Steroid Biochemistry and Molecular Biology, $117,50-55$.

Gasparini, J., Bize, P., Piault, R., Wakamatsu, K., Blount, J.D., Ducrest, A.L. \& Roulin, A. (2009) Strength and cost of an induced immune response are associated with a heritable melanin-based colour trait in female tawny owls. Journal of Animal Ecology, 78, 608-616.

Gelman, A., Hill, J. \& Yajima, M. (2012) Why we (usually) don't have to worry about multiple comparisons. Journal of Research on Educational Effectiveness, 5, 189-211.

Gelman, A., Su, Y.S., Yajima, M., Hill, J., Pittau, M.G., Kerman, J. \& Zheng, T. (2012) arm: data analysis using regression and multilevel/hierarchical models. $\mathrm{R}$ package version 1.5-03. Available at: http://cran. r-project.org/package $=$ arm/

Gonzalez, G., Sorci, G., Møller, A.P., Ninni, P., Haussy, C. \& De Lope, F. (1999) Immunocompetence and condition-dependent sexual advertisement in male house sparrows (Passer domesticus). Journal of Animal Ecology, 68, 1225-1234.

Groothuis, T. \& Schwabl, H. (2008) Hormone-mediated maternal effects in birds: mechanisms matter but what do we know of them? Philosophical Transactions of the Royal Society of London B: Biological Sciences, 363, $1647-1661$.

Henriksen, R., Groothuis, T.G. \& Rettenbacher, S. (2011) Elevated plasma corticosterone decreases yolk testosterone and progesterone in chickens: linking maternal stress and hormone-mediated maternal effects. PLoS ONE, 6, e23824

Henriksen, R., Rettenbacher, S. \& Groothuis, T. (2011) Prenatal stress in birds: pathways, effects, function and perspectives. Neuroscience and Biobehavioral Reviews, 35, 1484-1501.
Jenni-Eiermann, S., Hasselquist, D., Lindstrom, A., Koolhaas, A. \& Piersma, T. (2009) Are birds stressed during long-term flights? A wind-tunnel study on circulating corticosterone in the red knot. General and Comparative Endocrinology, 164, 101-106.

Jenny, M., Holzgang, O. \& Zbinden, N. (2005) Das Rebhuhn - Symbol für eine artenreiche Kulturlandschaft. Schweizerische Vogelwarte, Sempach.

Keller, L.F., Biebach, I., Ewing, S.R. \& Hoeck, P.E.A. (2012) The genetics of reintroductions: inbreeding and genetic drift. Reintroduction Biology: Integrating Science and Management (eds J.G. Ewen, D.P. Armstrong, K.A. Parker \& P.J. Seddon), pp. 360-394. John Wiley \& Sons, Chichester.

Kim, S., Noguera, J., Morales, J. \& Velando, A. (2010a) Heritability of resistance to oxidative stress in early life. Journal of Evolutionary Biology, 23, 769-775

Kim, S.Y., Velando, A., Sorci, G. \& Alonso-Alvarez, C. (2010b) Genetic correlation between resistance to oxidative stress and reproductive life span in a bird species. Evolution, 64, 852-857.

Kitaysky, A.S., Romano, M.D., Piatt, J.F., Wingfield, J.C. \& Kikuchi, M. (2005) The adrenocortical response of tufted puffin chicks to nutritional deficits. Hormones and Behavior, 47, 606-619.

Klein, S.L. (2000) The effects of hormones on sex differences in infection: from genes to behavior. Neuroscience and Biobehavioral Reviews, 24, 627-638.

Knight, J.A. (2000) Review: free radicals, antioxidants, and the immune system. Annals of Clinical and Laboratory Science, 30, 145-158.

Lesgards, J.F., Durand, P., Lassarre, M., Stocker, P., Lesgards, G., Lanteaume, A., Prost, M. \& Lehucher-Michel, M.P. (2002) Assessment of lifestyle effects on the overall antioxidant capacity of healthy subjects. Environmental Health Perspectives, 110, 479-486.

Levine, S. \& Ursin, H. (1991) What is stress? Stress. Neurobiology and Neuroendocrinology (eds M.R. Brown, G.F. Koob \& C. Rivier), pp. 3-21. Marcel Dekker, New York.

Love, O.P., McGowan, P.O. \& Sheriff, M.J. (2012) Maternal adversity and ecological stressors in natural populations: the role of stress axis programming in individuals, with implications for populations and communities. Functional Ecology, 27, 81-92.

Love, O.P. \& Williams, T.D. (2008a) The adaptive value of stress-induced phenotypes: effects of maternally derived corticosterone on sex-biased investment, cost of reproduction, and maternal fitness. The American Naturalist, 172, E135-E149.

Love, O.P. \& Williams, T.D. (2008b) Plasticity in the adrenocortical response of a free-living vertebrate: the role of pre- and post-natal developmental stress. Hormones and Behavior, 54, 496-505.

Love, O.P., Salvante, K.G., Dale, J. \& Williams, T.D. (2008) Sex specific variability in the immune system across life-history stages. The American Naturalist, 172, E99-E112.

Lynn, S.E., Breuner, C.W. \& Wingfield, J.C. (2003) Short-term fasting affects locomotor activity, corticosterone, and corticosterone binding globulin in a migratory songbird. Hormones and Behavior, 43, 150-157.

Lynn, S.E., Stamplis, T.B., Barrington, W.T., Weida, N. \& Hudak, C.A. (2010) Food, stress, and reproduction: short-term fasting alters endocrine physiology and reproductive behavior in the zebra finch. Hormones and Behavior, 58, 214-222.

Martin, L.B. (2009) Stress and immunity in wild vertebrates: timing is everything. General and Comparative Endocrinology, 163, 70-76.

Matson, K.D., Ricklefs, R.E. \& Klasing, K.C. (2005) A hemolysis-hemagglutination assay for characterizing constitutive innate humoral immunity in wild and domestic birds. Developmental and Comparative Immunology, 29, 275-286.

McDougall, P.T., Reale, D., Sol, D. \& Reader, S.M. (2006) Wildlife conservation and animal temperament: causes and consequences of evolutionary change for captive, reintroduced, and wild populations. Animal Conservation, 9, 39-48.

Merkling, T., Leclaire, S., Danchin, E., Lhuillier, E., Wagner, R.H., White, J., Hatch, S.A. \& Blanchard, P. (2012) Food availability and offspring sex in a monogamous seabird: insights from an experimental approach. Behavioral Ecology, 23, 751-758.

Merrill, L., Angelier, F., O’Loghlen, A.L., Rothstein, S.I. \& Wingfield, J.C. (2012) Sex-specific variation in brown-headed cowbird immunity following acute stress: a mechanistic approach. Oecologia, 170, 25-38.

Metcalfe, N.B. \& Alonso-Alvarez, C. (2010) Oxidative stress as a life-history constraint: the role of reactive oxygen species in shaping phenotypes from conception to death. Functional Ecology, 24, 984-996. 
Metcalfe, N.B. \& Monaghan, P. (2001) Compensation for a bad start: grow now, pay later? Trends in Ecology \& Evolution, 16, 254-260.

Monaghan, P., Metcalfe, N.B. \& Torres, R. (2009) Oxidative stress as a mediator of life history trade-offs: mechanisms, measurements and interpretation. Ecology Letters, 12, 75-92.

Mousseau, T.A. \& Fox, C.W. (1998) The adaptive significance of maternal effects. Trends in Ecology \& Evolution, 13, 403-407.

Mueller, C., Jenni-Eiermann, S., Blondel, J., Perret, P., Caro, S.P., Lambrechts, M.M. \& Jenni, L. (2007) Circulating corticosterone levels in breeding blue tits Parus caeruleus differ between island and mainland populations and between habitats. General and Comparative Endocrinology, 154, 128-136.

Mukhopadhyay, P., Rajesh, M., Yoshihiro, K., Hasko, G. \& Pacher, P. (2007) Simple quantitative detection of mitochondrial superoxide production in live cells. Biochemical and Biophysical Research Communications, 358, 203-208.

Munro, C. \& Stabenfeldt, G. (1984) Development of a microtitre plate enzyme-immunoassay for the determination of progesterone. Journal of Endocrinology, 101, 41-49.

Nunn, C.L., Lindenfors, P., Pursall, E.R. \& Rolff, J. (2009) On sexual dimorphism in immune function. Philosophical Transactions of the Royal Society of London B: Biological Sciences, 364, 61-69.

Olsson, M., Wilson, M., Isaksson, C., Uller, T. \& Mott, B. (2008) Carotenoid intake does not mediate a relationship between reactive oxygen species and bright colouration: experimental test in a lizard. Journal of Experimental Biology, 211, 1257-1261.

Perez de Heredia, F., Gomez-Martinez, S. \& Marcos, A. (2012) Obesity, inflammation and the immune system. The Proceedings of the Nutrition Society, 71, 332-338.

Price, E.O. (1999) Behavioral development in animals undergoing domestication. Applied Animal Behaviour Science, 65, 245-271.

R Development Core Team (2012) R: A Language and Environment for Statistical Computing. R Foundation for Statistical Computing, Vienna, Austria. Available at: http://www.r-project.org/.
Ricklefs, R.E. \& Wikelski, M. (2002) The physiology/life-history nexus. Trends in Ecology \& Evolution, 17, 462-468.

Rolff, J. (2002) Bateman's principle and immunity. Proceedings of the Royal Society of London B: Biological Sciences, 269, 867-872.

Sachdev, S. \& Davies, K.J. (2008) Production, detection, and adaptive responses to free radicals in exercise. Free Radical Biology and Medicine, 44, 215-223.

Sapolsky, R.M., Romero, L.M. \& Munck, A.U. (2000) How do glucocorticoids influence stress responses? Integrating permissive, suppressive, stimulatory, and preparative actions. Endocrine Reviews, 21, 55-89.

Schoech, S.J. \& Hahn, T.P. (2008) Latitude affects degree of advancement in laying by birds in response to food supplementation: a meta-analysis. Oecologia, 157, 369-376.

Stier, K.S., Almasi, B., Gasparini, J., Piault, R., Roulin, A. \& Jenni, L. (2009) Effects of corticosterone on innate and humoral immune functions and oxidative stress in barn owl nestlings. Journal of Experimental Biology, 212, 2084-2090.

Stocker, P., Lesgards, J.F., Vidal, N., Chalier, F. \& Prost, M. (2003) ESR study of a biological assay on whole blood: antioxidant efficiency of various vitamins. Biochimica et Biophysica Acta-General Subjects, $1621,1-8$

Tschirren, B., Rutstein, A.N., Postma, E., Mariette, M. \& Griffith, S.C. (2009) Short- and long-term consequences of early developmental conditions: a case study on wild and domesticated zebra finches. Journal of Evolutionary Biology, 22, 387-395.

Turrens, J.F. (1997) Superoxide production by the mitochondrial respiratory chain. Bioscience Reports, 17, 3-8.

Wingfield, J.C., Maney, D.L., Breuner, C.W., Jacobs, J.D., Lynn, S., Ramenofsky, M. \& Richardson, R.D. (1998) Ecological bases of hormone-behavior interactions: the "emergency life history stage". American Zoologist, 38, 191-206.

Received 11 October 2012; accepted 25 February 2013

Handling Editor: Kevin McGraw 